\title{
Composition and Relative Counting
}

\author{
Massimiliano CARRARA*, Giorgio LANDO ${ }^{\dagger}$
}

\begin{abstract}
According to the so-called strong variant of Composition as Identity (CAI), the Principle of Indiscernibility of Identicals can be extended to composition, by resorting to broadly Fregean relativizations of cardinality ascriptions. In this paper we analyze various ways in which this relativization could be achieved. According to one broad variety of relativization, cardinality ascriptions are about objects, while concepts occupy an additional argument place. It should be possible to paraphrase the cardinality ascriptions in plural logic and, as a consequence, relative counting requires the relativization either of quantifiers, or of identity, or of the is one of relation. However, some of these relativizations do not deliver the expected results, and others rely on problematic assumptions. In another broad variety of relativization, cardinality ascriptions are about concepts or sets. The most promising development of this approach is prima facie connected with a violation of the so-called Coreferentiality Constraint, according to which an identity statement is true only if its terms have the same referent. Moreover - even provided that the problem with coreferentiality can be fixed - the resulting analysis of cardinality ascriptions meets several difficulties.
\end{abstract}

\section{Introduction. Composition, Indiscernibility, and Cardinality Ascriptions}

Composition is an operation by which several things are unified into a single thing: it goes from many things to one thing. Therefore, its inputs and its output are different. This goes immediately against the intuition behind the so-called thesis of Composition as Identity (CAI), i.e. that composition is identity. A way of resisting this simple line of reasoning is to claim that "to be many", "to be one", as well as "to be three" and every other cardinality ascription, are relative.

Some backers of CAI think that this line of reasoning can be made to work, but the exact way in which this result could be accomplished is often left implicit. This paper aims to fill this gap, by analysing and evaluating several different ways to relativize cardinality ascriptions. We will show that some ways do not deliver the expected results, while others rest on controversial assumptions, that in some cases backfire on CAI.

First of all, let us recap what CAI is, decide which versions of CAI are our critical target, and explain why cardinality ascriptions matter for these versions.

${ }^{*}$ University of Padua, Italy; Email: massimiliano.carrara@unipd.it.

†University of L’Aquila, Italy; Email: giorgio.lando@univaq.it. 
Consider the following sentences:

(1) There is a cat, Mina, who is sleeping.

(2) There is a mouse, Gino, who is dancing.

Whoever asserts (1) is ontologically committed to Mina; whoever asserts (2) is ontologically committed to Gino. Suppose now that someone, in a mereological vein, asserts also:

(3) There is the sum of the mouse, Gino, and of the cat, Mina: Gina.

and then goes on to infer (4) from (1), (2), and (3):

(4) There are Gina, Gino, and Mina.

Is the ontological commitment to the sum Gina a further commitment, with respect to Mina and Gino? Notoriously David Lewis's answer is: no. ${ }^{1}$ The relation between Gino and Mina on one side, and Gina on the other would be significantly analogous to identity. And indeed, nothing could be considered more ontologically innocent than the request to accept something identical to things already accepted. If you are already committed to Cicero, the admission that there is Tully is no further ontological commitment. Analogously, Gina would not be a further ontological commitment over and above the commitment to Gino and Mina.

CAI is generally meant to also apply to the many-many relation (which would be preposterous to call "composition") between different slicings of the same whole. Gina is not only composed of Mina and Gino, but also of the cells in them. According to CAI, Mina and Gino on the one hand, and the cells on the other, are the same portion of reality, and the relation is as analogous to one-one identity as their relation with the whole (Gina) is. When - in what follows - we speak of composition we aim to include also these many-many relations between different slicings of a same whole.

According to Lewis himself, composition is not exactly like standard, one-one identity. In particular, the so-called Principle of Indiscernibility of Identicals or Leibniz's Law would not hold for composition: the sum, Gina, is discernible from the pets, Mina and Gino.

Why? There are many potential sources of discernibility, and in particular various kinds of collective predications, but in this essay we will focus on only one, mentioned by Lewis himself as the most evident case of discernibility between a whole and its parts: cardinality ascriptions.

Lewis writes:

What's true of the many is not exactly what is true of the one. After all they are many while it is one. (Lewis, 1991, p. 87)

In the example above, Mina and Gino are two, and not one: while Gina, their sum, is one, and not two. This seems to make Mina and Gino discernible from Gina.

As another example, consider Benelux: it is composed of the Netherlands, Belgium, and Luxembourg; if we take it to be a physical entity, it is also composed of a certain amount of

${ }^{1}$ See in particular (Lewis, 1991), p. 81. 
molecules. Thus, the relation between Benelux and the countries, the relation between Benelux and the molecules, and the relation between the countries and the molecules are assimilated by CAI to identity. But Benelux is one, while the Netherlands, Belgium, and Luxembourg are three, and the molecules are a very large number. Thus, they seem to be discernible.

But, if these relations are not governed by the same principles that govern one-one identity (such as Leibniz's Law), then, perhaps, the ontological innocence of mereology is not warranted. Thus, the case of Gina would not be on a par with the case in which, if we are already committed to Cicero, then Tully is not a new ontological commitment.

According to Sider for example, any defender of CAI who denies Leibniz's Law "would arouse the suspicion that their use of 'is identical to' does not really express identity". (Sider, 2007, p. 59)

Thus, more recently, other philosophers - Einar Bøhn, Aaron Cotnoir, Paul Hovda, and Megan Wallace ${ }^{2}$ - attempted to show that the sum and its parts (and different slicings of a same whole) are indiscernible. In the mind of these philosophers, Leibniz's Law holds for composition; indeed composition is an instance of identity. This variety of CAI is usually dubbed strong CAI, in opposition to Lewis's allegedly weak or moderate version, ${ }^{3}$ where the composition relation is just analogous to identity. In order to extend Leibniz's Law to composition, the backers of CAI need to analyze cardinality ascriptions in order to avoid the apparent forms of discernibility that ensue from them.

CAI has received many objections in the literature. They concern the grammaticality of many-one identity statements or other forms of discernibility between whole and parts. ${ }^{4}$ If you are persuaded by these objections, you will be perhaps unsurprised by the fact that CAI, when conjoined with other doctrines, delivers some counterintuitive and in some cases utterly undesirable results.

As a matter of fact, these objections have not stopped the debate about CAI, and the defenders of CAI have elaborated ingenious answers to the objections. A quick look at the growing literature on CAI reveals that it is common to resort to relative counting, with frequent references to Frege's treatment of cardinality ascriptions. The debate on cardinality ascriptions is special in the debate on CAI, because the defenders of CAI can in this case resort to an independently plausible idea, with a solid historical pedigree (it dates back to Frege): the idea that cardinality ascriptions are not primarily or exclusively about objects, but about concepts. Given the independent appeal of this idea, it is important to show, through an in-depth analysis of the various ways in which it has been and can be developed, that this idea is of no significant help to CAI.

In this paper, the focus is exclusively on strong CAI's attempts to relativize cardinality ascriptions to concepts in order to extend Leibniz's Law to composition. ${ }^{5}$

\footnotetext{
${ }^{2}$ (Bøhn, 2009), (Bøhn, 2014), (Cotnoir, 2013), (Hovda, 2005), (Wallace, 2009), (Wallace, 2011b).

${ }^{3}$ Moderate CAI has been recently endorsed and developed in (Bricker, 2015).

${ }^{4}$ Some important objections are set forth in (van Inwagen, 1994), (Yi, 1999), (McKay, 2006) (ch. 2) and (Yi, 2014).

${ }^{5}$ Among the approaches that aim to extend Leibniz's Law to composition, a very peculiar alternative for the analysis of cardinality ascriptions can be traced in (Wallace, 2011b, pp. 820-822), and will be not investigated in this paper. According to Wallace, cardinality ascriptions should be interpreted as metalinguistic ascriptions about identity statements; what is actually counted are variables in statements about parts and the allegedly identical whole they compose. Wallace's metalinguistic counting has nothing to do with Frege-like relativizations to concepts,
} 
Lewis's version of CAI does not represent such an attempt. Yet another variety of CAI originally formulated in (Baxter, 1988a) and (Baxter, 1988b), and recently developed in (Turner, 2014), and sometimes dubbed stronger ${ }^{6}$ - rejects Leibniz's Law in general, also for one-one identity, and provides independent reasons, concerning for example the theory of change, for this rejection. As a result, there is no need to explain away the violation of Leibniz's Law, by relativizing cardinality ascriptions.

By contrast, the defenders of strong CAI aim to be perfectly conservative about identity, and to show that composition cases are simply new, often unjustly disregarded instances of identity. And this is exactly what, according to our analysis, leads to trouble. At many points, we will see that the most promising ways in which cardinality ascriptions about parts and whole could be relativized are connected to non-standard views of identity. Thus, in the attempt to show that composition is on a par with standard identity from the viewpoint of indiscernibility, the defenders of strong CAI end up endorsing an analysis of cardinality ascriptions that works only if a revisionary view of identity is adopted. Thus, Sider's worry that "their use of 'is identical to' does not really express identity" could be retorted against them.

In this paper we do not aim to show that strong CAI - or even less CAI in general - fails. We simply aim to show that the relativization of cardinality ascriptions - if it is expected to make whole and parts, and different slicings of a same whole, indiscernible - is a narrow route for strong CAI. In its less disappointing varieties, the relativization requires a non-conservative stance about identity. If these varieties are adopted, strong CAI cannot be construed as a kind of Quinean conservatism about identity, that simply unveils new cases of the same old relation. It is a rather in-depth revisionism about identity, that insists on being conservative on Leibniz's Law, at the expense of other traits of identity. In particular, we will see that in some cases plural identity (usually taken as an unproblematic plural cousin of standard one-one identity) needs to be redefined in problematic ways; and that, in other cases, the usual, intimate link between identity and reference ends up being severed.

In order to better appreciate the differences between the varieties of CAI and identify our critical target, it is useful to focus on the following argument (Numerical Discernibility Argument, NDA), concerning the case of a whole $t$ and its three parts $u u^{7}$

There are two premisses:

(P1) $t$ is the sum of $u u$;

(P2) $u u$ are three and not one; $t$ is one and not three.

On the basis of the two premisses and taking into account Leibniz's Law, the following conclusion is inferred:

and raise different worries. For this reason, it will not be discussed in this paper.

(Wallace, 2011b) discusses also relative counting, and introduces metalinguistic counting as a tentative alternative to it. Wallace's motivations for seeking an alternative are different from our objections to relative counting. She is in particular worried by "the controversy over what exactly counts as sortals [...] and what does not" (p. 820). These concerns echo a criticism of CAI set forth in (Yi, 1999), see also §2.

${ }^{6}$ (Wallace, 2011a, p. 807).

${ }^{7}$ Following the predominant convention in plural logic, we use double letters as plural constants $(u u, v v, \ldots)$ and plural variables $(x x, y y, \ldots)$. 
(C) It is not the case that $t$ and $u u$ are identical.

Lewis accepts the argument NDA and its conclusion (C), at least if by "identity" we mean a relation governed by the principles that govern one-one identity. In Lewis's mind, composition has a lot in common with one-one identity, which would be enough to vindicate the ontological innocence of mereology; however, composition is not governed by the same principles. ${ }^{8}$ Also all the philosophers (such as (van Inwagen, 1994) and many others) who reject CAI would consider the argument as compelling proof that CAI is a false thesis.

Other versions of CAI reject the argument and its conclusion. Baxter rejects Leibniz's Law, and thus would argue that (C) does not follow from (P1) and (P2). But others - the defenders of strong CAI, our only critical target - hope to block the argument by rejecting (P2), and in particular by relativizing cardinality ascriptions to concepts. They resort to relative counting in order to deny that the whole and the parts differ in number and that they are, as a consequence, discernible.

But in what sense are cardinality ascriptions - in a broadly Fregean way - relative? Frege in his The Foundations of Arithmetic aimed to show that the real subjects of cardinality ascriptions are concepts, and not ordinary objects.

Frege's strategy was to prove this thesis by a reductio. Consider the following two passages:

While looking at one and the same external phenomenon, I can say with equal truth both "It is a copse" and "It is five trees", or both "Here are four companies" and "Here are 500 men". (Frege, 1950, §84)

An object to which I can with equal justice ascribe various numbers is not the actual bearer of a number. (ibid., §22)

For Frege this means that objects or phenomena are not the proper subjects of cardinality ascriptions. The proper subjects of cardinality ascriptions are instead certain kinds of concepts.

Thus, in the pars destruens of his argument, Frege claims that, apparently, one and the same object is the subject of various, incompatible cardinality ascriptions. But in the pars construens, he concludes that only some concepts are subjects of cardinality ascriptions. As a result, strong CAI can resort to Frege's relativization of cardinality ascriptions in two broad ways:

1. by insisting - contra Frege - that objects are the proper subjects of cardinality ascriptions;

2. by agreeing with Frege that objects are not the proper subjects of cardinality ascriptions: concepts or - as we will see - sets in some way connected to concepts are the proper subjects of cardinality ascriptions.

Both alternatives have been developed in various ways, and could be developed in others, to support strong CAI. We aim to analyze all the prima facie plausible varieties of both alternatives,

\footnotetext{
${ }^{8}$ It is not completely clear whether (Lewis, 1991) classifies composition as a kind of identity that does not respect Leibniz's Law, or if he thinks that composition is merely analogous to identity. While the latter interpretation is quite common in the literature, (Bøhn, 2011) argues convincingly in favour of the former.
} 
but, in order to also keep in touch with the current literature, when possible for each of the two broad strategies we focus on its currently most developed implementation. In the case of 1 ., we focus on the approach of (Bøhn, 2009) and (Bøhn, 2014); for 2., we consider the so-called theory of general identity of (Cotnoir, 2013)..$^{9}$ Incidentally, in the course of our analysis (and in particular in §§8-9) we will also discuss Frege's role in the debate on strong CAI.

The rest of the paper is organized as follows. In §§2-7 we review and criticize the first broad strategy of relativizing numerical ascriptions (1.), according to which their proper subjects are objects. In $\S 8-12$ we analyze the second broad strategy (2.), according to which the proper subjects of cardinality ascriptions are certain concepts or certain sets. Finally, in $§ 13$ we draw some conclusions.

\section{Counting Objects?}

According to the first broad approach, cardinality ascriptions are - contra Frege - about objects, even if they should be relativized nonetheless to concepts. One reason for preferring this route over the second could be the desire to minimize the ensuing semantic revisionism: the syntactic form of cardinality ascriptions in English and other natural languages suggests that they are about objects. A second reason (explicitly mentioned in (Bøhn, 2014, p. 145)) is that cardinality ascriptions are not the only obstacle in the way of strong CAI's attempt to extend indiscernibility to composition. For example, it seems that Benelux is the only member of its singleton $\{$ Benelux , while the Netherlands, Belgium, and Luxembourg are not members of \{Benelux\}. The Netherlands, Belgium, and Luxembourg are members of the European Union, while Benelux is not. Each of these cases can be and has been handled in the literature in various ways that lie beyond the scope of this paper. But, in general, a unified treatment is preferable: the proper subjects of cardinality ascriptions should also be the proper subjects of the other ascriptions that are potential sources of discernibility between a whole and its parts. And, in the case of the other potential sources, it seems absolutely clear that concepts are not the proper subjects: the Netherlands, Belgium, Luxembourg, and Benelux (and not some concept they fall under) are or are not members of sets and of the European Union.

If the proper subjects of cardinality ascriptions are objects, then concepts have to play a different role. Namely, they could occupy an additional argument place of numerical predicates (Bøhn, 2014, p. 146).

Thus, Benelux would actually be both one, and three, and a very large number, but each of these ascriptions should be relativized to a concept, which occupies an additional, hidden argument place. Benelux will be one from the viewpoint of the concept multinational entity; from this viewpoint, it is neither three nor a very large number. Benelux would be three from the viewpoint of the concept country; when conceptualized in this way, it is neither one nor a very large number. Benelux would also be a very large number (and neither one nor three) from the viewpoint of the concept molecule.

\footnotetext{
${ }^{9}$ Among the other supporters of strong CAI, (Hovda, 2005) does not really illustrate his approach to cardinality ascriptions. (Wallace, 2011b) distinguishes two approaches that she finds attractive: a kind of relative counting of objects very similar to that of (Bøhn, 2009) and the very different kind of metalinguistic approach we have mentioned and left aside in fn. 5. See fn. 13 about (Spencer, 2016).
} 
Molecules in Benelux would be three from the viewpoint of the concept country, and when we formalize such a statement we need to add an argument place for this concept ( $T$ is the unanalyzed predicate "are exactly three"; $m m$ is a plural term denoting the molecules; $c_{c}$ is a singular term for the concept country):

$$
T\left(m m, c_{c}\right)
$$

(Three Relativized, Unanalyzed)

Since concepts occupy argument places in the proper logical form of numerical ascriptions, no exception to Leibniz's Law is ever obtained. A formula of the form $F\left(x x, c_{1}\right) \wedge \neg F\left(x x, c_{2}\right)$ is no contradiction. ${ }^{10}$

(Yi, 2014) expressed some serious concerns about Bøhn's solution. How specific should the concepts be? Consider a square, that is composed of four smaller squares. According to strong CAI, the smaller squares are identical to the bigger square. Bøhn tells us that, in applying the numerical predicate "be four" to it/them, we should always add an argument place for a concept. But suppose that this concept is the concept square: is/are it/they 4 or 1 from the viewpoint of the concept square? Or perhaps 5? It could seem that different, incompatible numerical predicates could be applied to the whole and the parts, even once a concept is factored in. Some concepts (such as square in this case) allow for different ways of counting a single portion of reality. They deliver cases in which $c_{1}$ and $c_{2}$ are actually the same concept, and as a result a formula of the form $F\left(x x, c_{1}\right) \wedge \neg F\left(x x, c_{2}\right)$ is a contradiction.

However, Bøhn could simply reply that we should be more selective in choosing the concept that fills the additional argument place of the problematic predicates. ${ }^{11}$ The concept should not admit multiple subdivisions of one and the same portion of reality: at least in the specific case, the concept square is not suitable for the task, and we should perhaps choose instead the concept of being a square with a certain area. Suppose that the bigger square has an area of 4 $i n^{2}$, while each of the smaller squares has an area of $1 \mathrm{in}^{2}$. Then, the bigger square would be one when thought of as a square with an area of $4 \mathrm{in}^{2}$, and would be four when thought of as a square with an area of $1 \mathrm{in}^{2}$.

A clear criterion for identifying the right concepts should be provided, one that admits the concept square with an area of $4 \mathrm{in}^{2}$ and the concept square with an area of $1 \mathrm{in}^{2}$, but excludes the bare concept square.

The problems we will focus are quite independent of the issue of choosing the right concepts, and stem from the fact that numerical ascriptions are not unanalysable primitives, but have standard paraphrases.

\section{Cardinality Ascriptions: Logical Paraphrases, Non-Logical Paraphrases}

The paraphrases of cardinality ascriptions have the pivotal function of explaining the pervasive inferential links between them. For example, that some things are exactly three should imply

\footnotetext{
${ }^{10}$ Cfr. (Bøhn, 2014, p. 146).

${ }^{11}$ For the general problems concerning the concepts suitable for relative counting, see (Koslicki, 1997).
} 
that they are at least two, and that they are more than two, and less than seven, and so on.

The standard logical paraphrases of cardinality ascriptions manage to ground these kinds of inferences. These paraphrases can exploit set theory, or plural logic. We focus on plural logic, since it is the logical tool of reference in all the debates about strong CAI. However, nothing in our following analysis depends on the peculiarities of plural logic with respect to set theory.

The standard paraphrases of cardinality ascriptions in plural logic resort to identity, to the characteristic plural logic relation $<(\text { is one of })^{12}$, and to standard quantifiers and propositional connectives.

Let us consider the paraphrase of an absolute cardinality ascription, and then consider the impact of relativizations $a$ la Bøhn. To say that some things, denoted by the plural term $t$, are exactly three, is tantamount to saying that there is an $x$, there is a $y$, and there is a $z$ such that $x$, $y$, and $z$ are mutually non-identical and each of them is one of $t t$, and nothing else is one of $t$. Formally:

$\exists x \exists y \exists z(x \neq y \wedge y \neq z \wedge x \neq z \wedge x<t t \wedge y<t t \wedge z<t t \wedge \forall w(w<t t \rightarrow w=x \vee w=y \vee w=z))$

(Three Logical Form)

The following claim that the $t t$ are at least two will follow from (Three Logical Form) by sheer logic:

$$
\exists x \exists y(x \neq y \wedge x<t t \wedge y<t t)
$$

All similar implications will be analogously warranted by logic.

But, according to the variety of strong CAI we are discussing, numerical ascriptions are not absolute, and have an additional argument position for a concept. Consider, again, the above claim that the molecules in Benelux are exactly three from the viewpoint of the concept country.

$$
T\left(m m, c_{c}\right)
$$

(Three Relativized, Unanalyzed)

It should imply that the molecules are, when thought of as countries, at least two, less than five, and so on. In order to ground these inferences, (Three Relativized, Unanalyzed) should be paraphrasable in the language of plural logic.

But how? Obviously, the paraphrase of the claim that $\mathrm{mm}$, when thought of as countries, are exactly three cannot also be the paraphrase of the claim that $\mathrm{mm}$, when thought of as molecules, are exactly three. These two claims are different: in the specific case, the first claim is expected to be true, while the second false.

\footnotetext{
${ }^{12}$ It is controversial that is one of is the fundamental relation in plural logic. For example, according to (Oliver and Smiley, 2013), the fundamental relation of plural logic is the many-many relation of is/are among. We will show in $\S 4$ that the relation of inclusion employed in cardinality ascription is is one of.
} 
Also the implications should be restricted to the same concept: the claim that $\mathrm{mm}$ are exactly three when thought of as countries should imply that $\mathrm{mm}$ are at least two when thought of as countries, but should not imply that $\mathrm{mm}$ are at least two when thought of as multinational entities.

We need to find a place for the concept in (Three Logical Form). But where ${ }^{13}$ If we look at (Bøhn, 2014) or (Bøhn, 2009), there is no explicit indication. In the following sections (§§4-6) we investigate different ways of "relativizing" (Three Logical Form) to a concept.

However, before investigating how the relativization of cardinality ascriptions could be mirrored in their plural logic paraphrases, we have to discuss two alternatives that, according to us, do not really make a difference. The first alternative (Contextual parameters below) concerns the proper role of concepts in the semantics of cardinality ascriptions. The second (Mereological paraphrases) concerns the hypothesis of radical departures from the standard paraphrases, in which $\prec$ (or its set-theoretical corresponding $\in$ ) would not appear at all.

Contextual parameters. A supporter of strong CAI could claim that concepts play a role in the semantic evaluation of cardinality ascriptions, instead of occupying hidden, syntactic argument places of numerical predicates, as in Bøhn's proposal.

Concepts could be evaluation parameters supplied by the context. The claims "Benelux is one" and "The Netherlands, Belgium, and Luxembourg are not one" would be made compatible, but not by adding an argument place to the predicates. Instead, the semantics would be such that numerical ascriptions should always be evaluated according to a certain concept. "Benelux is one" would be true when evaluated from the viewpoint of the concept multinational entity, and false when evaluated from the viewpoint of the concept

\footnotetext{
${ }^{13}$ (Spencer, 2016) (\$3) doubts that CAI is forced to provide relativized logical paraphrases of cardinality ascriptions. By contrast, he argues that cardinality ascriptions should not be analyzed in logical terms at all, but should be considered as irreducibly primitive. As a consequence, the logical paraphrases of cardinality ascriptions could not be used as an argument against CAI. Spencer's train of thought follows (Salmon, 1997)'s discussion of cardinality ascriptions that include fractional or negative numbers - such as "the oranges are two and a half", or "the dollars in my bank account are -2". According to Salmon (and more recently (Liebesman, 2015, 2016)) cardinality ascriptions in the above cases cannot be translated in logical terms. The unavailability of the logical paraphrase in these cases would empty the motivation for providing logical paraphrases of cardinality ascriptions in general. Indeed, the usual motivation for providing such paraphrases is the need to explain in a unified way the inferences between different cardinality ascriptions, such as when "the oranges on the table are exactly three" entails "the oranges on the tables are at least two". These inferences seem to concern also the fractional cases: from "the oranges on the table are exactly one and a half" it follows that "the oranges on the table are at least one". Insofar as there is no logical paraphrase in these cases, it would not be possible to explain all the inferences among cardinality ascriptions through logical paraphrases. If we cannot explain all these inferences, logical paraphrases lose their explanatory value, also for non-fractional, non-negative cardinality ascriptions. Thus, the defender of CAI could say that there is no need to go beyond (Three Relativized, Unanalyzed) in the relativization, and no need to ask (as we are asking) what should be relativized in logical paraphrases such as (Three Logical Form).

Our tentative reply is that - no matter if they are always available or explicative - logical paraphrases are sometimes available, and that, in particular for all the non-fractional and non-negative cases, they deliver the right truth conditions: this is enough to force the defender of CAI to decide what she wants to relativize in the logical paraphrase. It does not matter whether the paraphrase is explicative with respect to (Three Relativized, Unanalyzed). Nonetheless, we acknowledge that the relation between fractional counting, negative counting and CAI is complex and underexplored. We plan to discuss Spencer's interesting approach in another paper we are working on.
} 
country; and the same would hold for "The Netherlands, Belgium and Luxembourg are one". The fact that there are things and a number $n$ such that it is true (given a certain contextual parameter) that these things are $n$ and it is true (given a different conceptual parameter) that these same things are not $n$ would not be a counterexample to Leibniz's Law. Analogously, the fact that "it rains" is true under certain temporal and spatial parameters, and false under other spatial and temporal parameters is not an exception to Leibniz's Law.

The distinction between taking the concept as occupying an additional argument place and taking it as a contextual parameter has no specific bearing on the problems we are going to discuss. It could be very important when assessing the linguistic credibility of relative counting in general, ${ }^{14}$ but does not dispel, or substantially transform, the need to relativize the logical paraphrase of cardinality ascriptions to concepts, upon which our analysis will hinge.

Indeed, the need to relativize would simply be transferred: instead of looking for a syntactic place for concepts in the logical paraphrase, we should look for the subformulas of the logical paraphrase whose evaluation should be contextually parametrized to concepts.

In the next sections, we will consider the hypothesis that various elements of the vocabulary of (Three Logical Form) (quantifiers, identity, $<$ ) should be relativized to concepts, and concepts will appear explicitly in the corresponding subformulas of (Three Logical Form). By contrast, if concepts were instead expected to act as contextual parameters, we should consider the hypothesis that the evaluation of subformulas of (Three Logical Form), which include these same elements of the vocabulary, should be contextually parametrized to a concept.

One cannot simply declare that the evaluation of (Three Logical Form) should be parametrized to a concept, without specifying what, in the vocabulary of (Three Logical Form), determines this need. As a result, there is a correspondence between additions of syntactic places in certain subformulas and parametrized evaluations of these same subformulas, without any deep, specific reason to prefer one route over the other.

In the next section, we focus on the first option (the syntactic relativization), both because Bøhn explicitly endorses it, ${ }^{15}$ and because it makes more vivid where in the logical paraphrase the relativization is at work.

Mereological paraphrases. A backer of strong CAI could look for a relativized paraphrase of numerical ascriptions that does not employ any specific predicate of plural logic or set-theory (for the sake of brevity, we will omit the set-theoretic alternative henceforth).

The most attractive way could be to use the mereological lexicon. To claim that $t t$ are three from the viewpoint of a concept $c$ would be to claim that the fusion of $t t(\Sigma(t t))$

\footnotetext{
${ }^{14}$ For a general discussion of the difference between argument places and contextual parameters (in the cases of space and time), see (King, 2003).

${ }^{15} \mathrm{~B} ø \mathrm{hn}$ does not discuss the hypothesis of contextual parameters at all. The indexical variant of (Cotnoir, 2013)'s treatment of cardinality ascriptions is a kind of contextual parametrization. We will discuss it in $§ 12$.
} 
has three parts that fall under the concept $c$ (in the following formula, $P$ is mereological parthood):

\footnotetext{
$\exists x \exists y \exists z(x$ falls under $c \wedge y$ falls under $c \wedge z$ falls under $c \wedge$

$\wedge x \neq y \wedge y \neq z \wedge x \neq z \wedge x P \Sigma(t t) \wedge y P \Sigma(t t) \wedge z P \Sigma(t t) \wedge \quad$ (Three Mereological)

$\wedge \forall w(w$ falls under $c \wedge w P \Sigma(t t) \rightarrow w=x \vee w=y \vee w=z))$
}

What would be actually counted in this case would be the fusion, and, trivially, given a whole and its parts, the fusion of the whole is the whole itself, which is also the fusion of its parts. Given that we have decided to leave aside the problems of choosing the right concepts, no decisive objection against the soundness of this mereological paraphrase comes to our mind.

One could try to object that (Three Mereological) would not be a logical paraphrase, because $\Sigma$ and $P$ would not belong to the logical vocabulary. But some backers of strong CAI (including (Bøhn, 2014)) would probably insist that CAI itself makes the mereological notions as logical as identity is. Thus, it would be arguably unfair to presuppose that mereology is not logic, when discussing the possible paraphrases of cardinality ascriptions which strong CAI could adopt. ${ }^{16}$

The reason why this mereological paraphrase does not solve any problem is different, and consists simply in the fact that, even if (Three Mereological) were licensed as an acceptable paraphrase of (Three Relativized, Unanalyzed), the standard paraphrase (Three Logical Form) would be still there. The resort to the mereological paraphrase would not explain why the standard paraphrase would be unviable. If $<$ is a predicate in our vocabulary, then (Three Logical Form) is a well-formed sentence of our language, which - outside of the debate about CAI - is usually taken to be the right paraphrase. Now, why should the availability of another allegedly right paraphrase discredit the standard one? We are unable to imagine how the adoption of (Three Mereological) could motivate the thesis that it is impossible to paraphrase soundly (Three Relativized, Unanalyzed) in terms of $\prec$.

If it is possible to paraphrase (Three Relativized, Unanalyzed) in terms of $\prec$, then this paraphrase should not make the parts discernible from the whole. Otherwise, it does not matter that there is another paraphrase at disposal, such as (Three Mereological), that does not make them discernible. One sound paraphrase that makes (say) the Netherlands, Belgium, and Luxembourg discernible from Benelux is enough to get strong CAI in trouble. The availability of other paraphrases does not alleviate this trouble.

Thus, in general, the eventual availability of other paraphrases (in mereological, or in other, eventually non-logical terms) does not solve the problem of relativizing the standard, logical paraphrases. And, now, we turn to appraise various ways in which this relativization could be realized.

\footnotetext{
${ }^{16}$ See (Lando, 2017, ch. 3 and ch. 10) for a discussion of the alleged logicality of mereology.
} 


\section{Restricting Quantifiers, Relativizing Quantifiers, Relativizing Referential Expressions}

The simplest strategy would be to make clear, within (Three Logical Form), that the things we are speaking about fall under a certain concept $c$. This could be done either by restricting all the quantifiers, or by relativizing them, or by relativizing all the referential expressions in (Three Logical Form).

Let us begin with the restriction of quantifiers. The restriction could resort to the above relational predicate falls under, but it is convenient to introduce, for each concept (say: the concept country, $c_{c}$ ), a corresponding predicate $C$ ("be a country").

$$
\forall x\left(C x \leftrightarrow x \text { falls under } c_{c}\right)
$$

We obtain the following paraphrase of the sentence "the Netherlands, Belgium, and Luxembourg are three" ( $t t$ is here a plural constant denoting the Netherlands, Belgium, and Luxembourg):

$$
\begin{array}{r}
\exists x \exists y \exists z(C x \wedge C y \wedge C z \wedge x \neq y \wedge y \neq z \wedge x \neq z \wedge x<t t \wedge y<t t \wedge z<t t \wedge \\
\wedge \forall w(C w \wedge w<t t \rightarrow w=x \vee w=y \vee w=z))
\end{array}
$$

(Three with Restricted Quantifiers)

This paraphrase implies that $t t$ are at least three absolutely, independently of any concept:

$$
\exists x \exists y \exists z(x \neq y \wedge y \neq z \wedge x \neq z \wedge x<t t \wedge y<t t \wedge z<t t \text { ) (At Least Three Absolutely) }
$$

This would mean that, if $t t$ (the Netherlands, Belgium, and Luxembourg) are exactly one when thought of as a multinational entity, then they are at least one tout court. And if they are a very large number when thought of as molecules, then they are at least that very large number tout court. This outcome seems surprising: it would seem that the Netherlands, Belgium, and Luxembourg are not a very large number in any absolute sense.

The introduction of concepts in numerical ascriptions has the purpose, from the viewpoint of CAI, to make acceptable some prima facie unacceptable numerical ascriptions, such as "the Netherlands, Belgium, and Luxembourg are at least a very large number". But - the defender of CAI argues - "the Netherlands, Belgium, and Luxembourg are at least a very large number" becomes acceptable if we factor in a concept: from the viewpoint of the concept molecule, perhaps the Netherlands, Belgium, and Luxembourg really are at least a very large number. However, the logical paraphrases of cardinality ascriptions should not be such that it is so easy to eliminate concepts in them, because, once the concepts are eliminated, the resulting 
absolute, concept-free cardinality ascription risks being as implausible as the original, prima facie unacceptable cardinality ascription was. This would happen in the inference from the logical paraphrase of "the Netherlands, Belgium, and Luxembourg are at least a very large number (when thought of as molecules)" to the logical paraphrase of "the Netherlands, Belgium, and Luxembourg are at least a very large number (absolutely)" (and this inference is as logically trivial as the inference from (Three with Restricted Quantifiers) to (At Least Three Absolutely)).

Thus, a first problem for the strategy of restricting quantifiers is that on one hand it aims to make sense of some implausible, non-relativized cardinality ascriptions, by relativizing them to concepts; on the other, the resulting logical paraphrases of cardinality ascriptions imply some of those same, implausible, non-relativized cardinality ascriptions, such as the claim that the Netherlands, Belgium, and Luxembourg are at least a very large number absolutely. ${ }^{17}$

This perplexing outcome could be avoided if, instead of restricting the quantification through a predicate such as $C$, we relativize the quantifiers themselves, i.e. we introduce specific quantifiers for entities falling under a certain concept. $\forall_{c}$ and $\exists_{c}$ would be the quantifiers for countries. "The Netherlands, Belgium, and Luxembourg are three" would be paraphrased as follows:

$$
\begin{array}{r}
\exists_{c} x \exists_{c} y \exists_{c} z(x \neq y \wedge y \neq z \wedge x \neq z \wedge x<t t \wedge y<t t \wedge z<t t \wedge \\
\left.\wedge \forall_{c} w(w<t t \rightarrow w=x \vee w=y \vee w=z)\right)
\end{array}
$$

(Three with Relativized Quantifiers)

The relativized quantifiers should receive an appropriate inferential characterization, otherwise (Three with Relativized Quantifiers) would come up against the same issue affecting the restricted quantifiers (namely, the cardinality ascriptions with restricted quantifiers would imply cardinality ascriptions with unrestricted quantifiers). For example, a restricted existential quantification should not imply an absolute existential quantification. Perhaps, in such a scenario, absolute quantification could be dispensed with altogether. In any case, the expected outcome is that the above claim that $t t$ are at least three tout court should not follow from (Three with Relativized Quantifiers).

This would require plenty of quantifiers: one for each concept involved in counting (thus, perhaps, also for specific concepts such as square with a $1 \mathrm{in}^{2}$ size).

But there is a bigger problem, which concerns both the attempt to restrict quantifiers and the attempt to relativize them. This affects specifically the purpose of these attempts in the present context: Bøhn, together with the other backers of strong CAI, wants to show that the parts and the whole are, despite appearances, indiscernible. This means that - in non-opaque contexts - a name for the whole and a name for the parts should be mutually substitutable salva veritate. In (Three Relativized, Unanalyzed), a singular term for Benelux, or a plural term for the molecules in Benelux should be substitutable salva veritate for the term $t t$ denoting the

\footnotetext{
${ }^{17}$ One could try to make sense of absolute cardinality ascriptions such as by saying that to count absolutely is to count atomic parts. We will discuss the potential role of atoms in counting with reference to (Cotnoir, 2013) in $\S \S 10-12$.
} 
Netherlands, Belgium, and Luxembourg. However it is divided up, that portion of reality is 3 when thought of as countries.

But neither in (Three with Relativized Quantifiers) nor in (Three with Restricted Quantifiers) can $t t$ be replaced salva veritate by a term for Benelux or by a term for molecules, such as $\mathrm{mm}$. In the case of $\mathrm{mm}$, there are not three different countries each of which is one of $\mathrm{mm}$. No country is one of the molecules in Benelux. No non-molecule is one of the molecules.

Since we are employing only the logical language, it would be arbitrary and unsupported to attribute the failure of substitution to the opacity of the context. The defender of CAI could remark that it is the definite description "the molecules" (or "the molecules in Benelux") that triggers a negative evaluation of the claim that, for example, Benelux is one of the molecules; and that, by contrast, the non-descriptive constant $m m$ does not elicit a similarly clear evaluation. In the typical jargon of CAI, the countries are one of what $\mathrm{mm}$ designates, because what $\mathrm{mm}$ designates is a portion of reality.

The problem is that $\mathrm{mm}$ is expected to be coreferential with a description such as "the molecules in Benelux". Thus, the former and the latter should be mutually replaceable salva veritate in non-opaque contexts. Moreover, definite descriptions could be added in a formal language. Let $M B$ be the predicate "be a molecule in Benelux". Then $m m$ should be coreferential with the plural definite description $\iota x x(M B x x)$. Thus, as much as no country is one of $\iota x x(M B x x)$, no country is one of $\mathrm{mm}$. It does not matter that $\mathrm{mm}$ is a direct, non-descriptive referential expression, insofar as it is coreferential with a definite description such as $\operatorname{lx} x(M B x x)$ or "the molecules in Benelux".

Nonetheless, one could try to develop in another direction the impression that the problem lies in the referential expressions such as $\mathrm{mm}$. Perhaps, when you relativize a claim about some things to a concept, also the way in which we refer to these things should be relativized to that concept. One could envisage a language in which all the referential expressions are relativized. The relativization of the variables would replace the relativization of the quantifiers we attempted in (Three with Relativized Quantifiers), and the significant novelty would be that the constants for the counted entities would be relativized too.

"The Netherlands, Belgium, and Luxembourg are three" would be paraphrased as follows:

$$
\begin{array}{r}
\exists x \exists y \exists z\left(x_{c} \neq y_{c} \wedge y_{c} \neq z_{c} \wedge x_{c} \neq z_{c} \wedge x_{c}<t t_{c} \wedge y_{c}<t t_{c} \wedge z_{c}<t t_{c} \wedge\right. \\
\left.\wedge \forall w\left(w_{c}<t t_{c} \rightarrow w_{c}=x_{c} \vee w_{c}=y_{c} \vee w_{c}=z_{c}\right)\right)
\end{array}
$$

(Three with Relativized Referential Expressions)

It is far from clear what happens if we now try to replace $t t_{c}$ (a plural term denoting the Netherlands, Belgium, and Luxembourg qua countries) with a term for Benelux, or for the molecules. $t t_{c}$ cannot be replaced salva veritate by a term for Benelux or for the molecules relativized to another concept - say the concept multinational entity, or the concept molecules. Suppose that, instead, we replace $t t_{c}$ with a term for Benelux relativized to the same concept, the concept country: $b_{c}$.

In this case, our semantic intuitions vacillate. On one side, $b_{c}$ denotes Benelux, and, insofar as Benelux is not a country, it would seem that no country can be one of Benelux, no matter 
the concept from the viewpoint of which we look at Benelux. However, the defenders of strong CAI could try to say that the relativizing concept enforces a certain way of dividing up the Benelux portion of reality, so that, for example, Belgium-qua-country really is one of Benelux-qua-country.

The semantic and metaphysical problems of such a form of qua-reference are beyond the purpose of this paper. However, it is clear that this line of thought leads to a deeply non-standard theory of identity. In the context of the logical paraphrases of cardinality ascriptions such as (Three with Relativized Referential Expressions), terms such as $b_{c}$ and $t t_{c}$ could perhaps be substitutable salva veritate. By contrast, the substitution of $b_{c}$ with terms such as $b_{m e}$ ("Beneluxqua-multinational entity") or $b_{m}$ ("Benelux-qua-molecules") would not be salva veritate. Indeed one and the same thing (for example Belgium-qua-country) would be one of $b_{c}$, but not one of $b_{m e}$ or $b_{m}$. However, at least within the limits of a standard theory of identity, $b_{c}$ is expected to be identical both to $b_{m e}$ and to $b_{m}$. Thus, the problem with Leibniz's Law would be simply moved from the identities between whole and parts conceptualized in the same way, to the identities between things conceptualized in different ways.

In other words, after this move, we would still have cases of identity without indiscernibility, in contrast with strong CAI's claim to be perfectly conservative about identity, i.e. to simply extend to composition the standard identity relation (essentially characterized by Leibniz's Law). What would happen is that, in the struggle of extending Leibniz's Law to composition, the defenders of CAI would violate Leibniz's Law for other cases of identity relations. In these cases one and the same object would be designated under the viewpoint of different concepts. Thus the relativization of referential expressions does not serve the cause of strong CAI any better than the restriction or the relativization of quantifiers we discussed above.

A last attempt to save the general kind of approach discussed in this section could be to focus on the interpretation of $<$ in (Three with Restricted Quantifiers) or (Three with Relativized Quantifiers).

In plural logic, there are two main relations of inclusion. One of them - the one we have expressed above with $\prec$ and we will focus on also in the next sections - is usually read as is one of, and is a one-many relation between one entity and a plurality of entities the entity belongs to. It corresponds to set-theoretic membership $\epsilon$, in the sense that $x$ is one of $(<)$ yy iff $x$ is a member of $(\in)$ the set of the $y y$. The other inclusion relation - usually read is/are among and expressed with a variety of symbols, for example $\lesssim-$ is a many-many relation, and it is definable in terms of is one of: $x x$ are among $(\precsim)$ yy iff every $z$ that is one of $(<) x x$ is also one of $(<)$ yy. $\lesssim$ corresponds to the set-theoretic relation of being a subset $\subseteq: x x$ are among $(\lessgtr)$ yy iff the set of $x x$ is a subset $(\subseteq)$ of the set of $y y{ }^{18}$

Now, the defender of CAI could try to argue that some of our evaluations of cardinality ascriptions crucially depend on focusing on $\prec$ instead of $\lesssim$. For example, we argued above that, if we replace in (Three with Restricted Quantifiers) $t t$, a term for the Netherlands, Belgium, and Luxembourg, with $\mathrm{mm}$, a term for the molecules, we obtain the false claim that there are three countries such that each of them is one of $\mathrm{mm}$, and no other country is one of $\mathrm{mm}$. The claim

\footnotetext{
${ }^{18}$ About the distinction between is one of and is/are among see (Rayo, 2002), (Yi, 2005) (p. 486), (McKay, 2006) (ch. 6). See (Oliver and Smiley, 2013) (p. 109) for a table about the different ways of interpreting and symbolizing inclusion relations in plural logic. The symbols we use are those of (Rayo, 2002) and (Linnebo, 2014).
} 
we would obtain is:

$$
\begin{array}{r}
\exists x \exists y \exists z(C x \wedge C y \wedge C z \wedge x \neq y \wedge y \neq z \wedge x \neq z \wedge x<m m \wedge y<m m \wedge z<m m \wedge \\
\wedge \forall w(C w \wedge w<m m \rightarrow w=x \vee w=y \vee w=z))
\end{array}
$$

However, suppose that (Three with Restricted Quantifiers) is formulated with $\lesssim$ instead of $\prec$. In that case, the claim we would obtain is:

$$
\begin{array}{r}
\exists x \exists y \exists z(C x \wedge C y \wedge C z \wedge x \neq y \wedge y \neq z \wedge x \neq z \wedge x \lesssim m m \wedge y \lesssim m m \wedge z \lesssim m m \wedge \\
\wedge \forall w(C w \wedge w \lesssim m m \rightarrow w=x \vee w=y \vee w=z))
\end{array}
$$

(Three with is/are among)

In evaluating this sentence, the defender of CAI might venture in a semantic hypothesis according to which a country such as Belgium, inasmuch as it is - according to CAI - identical to some molecules, can be among the molecules in Benelux. The sentence would be, as a consequence, true. We do not know what to think about the kind of semantic revisionism that ensues, but there is no need to worry about it, because it is wrong to paraphrase a cardinality ascription in the form of (Three with is/are among). ${ }^{19}$

Let us see why. Suppose that (Three with is/are among) is a kind of sentence in which, thanks to some deviant semantics, $t t$ and $m m$ can be substituted salva veritate. However, (Three with is/are among) is not a cardinality ascription, insofar as the variables are allowed to have as values many things, such as the molecules in Belgium that would be among the molecules in Benelux in the interpretation of (Three with is/are among) we sketched above. It is not a cardinality ascription because the things that are said to be a country in each of the three conjuncts $C x, C y$, and $C z$ are allowed to be more than one, and the sentence does not say how many they are. The fact that $<$ (and not $\lesssim$ ) is the relation of inclusion that should be used in cardinality ascriptions is confirmed by their set-theoretic counterparts. The typical paraphrase of a cardinality ascription in set-theoretic terms employs the relation $\epsilon$ of being an element, and not the relation $\subseteq$ of being $a$ subset.

Thus, (Three with is/are among) is not a cardinality ascription, and, as a consequence, is not a paraphrase of (Three Relativized, Unanalyzed). On the other hand, as we have seen, if either (Three with Relativized Quantifiers), or (Three with Restricted Quantifiers) were the sound logical paraphrase of (Three Relativized, Unanalyzed), then they would offer a clear case of discernibility between parts and whole, and between various partitions of the same whole. Finally, if the complex route of (Three with Relativized Referential Expressions) were chosen, then Leibniz's Law would be violated as a side effect in other cases. The backer of strong CAI should leave quantifiers and referential expressions alone and try to relativize something else.

\footnotetext{
${ }^{19}$ Given that $\lesssim$ and $\prec$ are interdefinable, there will be a much more complex way of paraphrasing (Three with Restricted Quantifiers) in terms of $\lesssim$, but this more complex paraphrase would be of no help in resolving the failure of substitution in (Three with Restricted Quantifiers).
} 


\section{Relativizing Identity}

The hypothesis of relativizing identity may seem attractive at first glance for strong CAI, once strong CAI has endorsed relative counting. When we conceptualize Benelux as a multinational entity, we do not make distinctions in it. When we apply the concept country to Benelux, we are ready to distinguish more than one thing (namely, three) in it. When we apply the concept molecule to it, we distinguish a lot of things in it. What changes from one conceptualization to another seems to be the way in which we distinguish things. One would suspect that we are using different notions of identity. Then, perhaps, identity itself should be relativized to the concept to which we relativize the unanalyzed cardinality ascription. ${ }^{20}$

This would mean that in (Three Logical Form) the claims of diversity between the three countries should negate a country-specific kind of identity. (Three Logical Form) should be paraphrased as follows:

$$
\begin{array}{r}
\exists x \exists y \exists z\left(\neg x==_{c} y \wedge \neg y==_{c} z \wedge \neg x={ }_{c} z \wedge x<t t \wedge y<t t \wedge z<t t \wedge\right. \\
\left.\wedge \forall w\left(w<t t \rightarrow w==_{c} x \vee w==_{c} y \vee w==_{c} z\right)\right)
\end{array}
$$

(Three with Relativized Identity)

It is worth noting that Bøhn rejects the relativization of identity to concepts: "Identity itself is of course not thus relational. Relative identity is worse than death". (Bøhn, 2014, p. 146, n. 10). ${ }^{21}$ While Bøhn does not explain his distaste for relative identity, it is not difficult to understand why relative identity does not satisfy the needs of strong CAI.

The doctrine of relative identity is difficult and controversial in itself. More specifically, it would be incoherent to appeal to relative identity in order to extend the (absolute and unrestricted) indiscernibility of identicals to composition, in the context of the defence of strong CAI. As we have already observed, the purpose of strong CAI is to show that the relations between whole and parts - and between different slicings of the same whole - are instances of the single, perfectly standard relation of identity, exhaustively characterized by reflexivity and Leibniz's Law. In the struggle of showing that composition is identity, the defenders of strong CAI should not surreptitiously embrace a doctrine of identity that runs counter the principles of standard identity.

The doctrine of relative identity holds that there are different identity relations, and no absolute one, and is therefore forced to relativize Leibniz's Law. Two things may be identical

\footnotetext{
${ }^{20}$ In general, one could hope to argue for relativizing identity on the basis of the general links between identity and cardinality, as they are expounded in (Geach, 1967) and (Alston and Bennett, 1984). Consider, for example, the sentence: "If $x$ is not $y$, then they are two". It would be indeed strange if the relativization concerned only the consequent, and not the antecedent: if relativization does affect cardinality, then it should also affect identity. However, (Blanchette, 1999) and (Carrara and Sacchi, 2007) have shown that the reasons why cardinality ascriptions can be deemed incomplete (and should therefore be relativized) do not translate into reasons to deem identity statements incomplete (and thus to relativize them).

${ }^{21}$ Also (Wallace, 2011b, n. 11), in her discussion of relative cardinality in the context of strong CAI, rejects any link with the doctrine of relative identity.
} 
from the viewpoint of a certain identity relation (say: $=_{c 1}$, relativized to a concept $c_{1}$ ), but different from the viewpoint of another identity relation (say: $=_{c 2}$, relativized to $c_{2}$ ). And, if $d e$ facto $x={ }_{c 1} y$ and $\neg x={ }_{c 2} y, x$ is identical to $y$ from the viewpoint of $=_{c 1}$, but at the same time discernible from $y$ (for, while $x$ is not identical to $y$ from the viewpoint of $=_{c 2}, y$ is identical to $y$ from the viewpoint of $=_{c 2}$ ). Perhaps, in a different perspective, one could try to combine a different variety of CAI with relative identity: no objection in this paper is aimed at this future attempt. By contrast, our purpose is to show that it is not possible to combine CAI with a canonical view of identity - as it is done in strong CAI - and the doctrine of relative identity obviously is not a canonical view of identity.

Relative identity, even if not worse than death, is in sharp contrast with the motivations leading philosophers such as Bøhn, Wallace, or Cotnoir to endorse strong CAI: according to strong CAI, composition is absolute identity and respects Leibniz's Law, but relative identity does not admit absolute identity and absolute indiscernibility.

Moreover, the relativization of identity fails to show that the whole and the parts are indiscernible. In fact, it shares a fatal defect with the restriction or relativization of quantifiers discussed in §4: in (Three with Relativized Identity) we cannot replace salva veritate $t$ with a singular term for Benelux, or with a plural term for the molecules in that portion of reality. There are not three country-like-different things each of which is one of the molecules.

Also in this case, nothing suggests that some kind of referential opacity is to blame, inasmuch as only logical vocabulary is employed. But, in this case, the failure of substitutivity is unsurprising: it can be explained by the above mentioned fact that the doctrine of relative identity should not expect relative identicals to be absolutely indiscernible. A consistent adopter of relative identity rejects absolute indiscernibility. Once absolute indiscernibility has been dropped, there is no reason why the terms for relative identicals should be expected to be replaceable salva veritate in every context. Again: if we are ready to relativize identity, there is no reason to insist on strong CAI. Various kinds of discernibility and failures of substitutivity are only to be expected. If you aim to extend Leibniz's Law to composition, you should avoid (Three with Relativized Identity), and try another option.

\section{Relativizing Is One of}

The last available option is to relativize is one of. We have seen in the two previous sections that it does not help to operate on quantifiers and identity. A defect shared by all the kinds of relativizations and restrictions discussed so far is that the resulting logical analyses of numerical ascriptions end up making the parts and the whole, or different partitions of the same whole discernible, in contrast with the most basic desideratum of strong CAI. The source of discernibility is that, given a term such as $t t$, what is one of $t t$ is not also one of $s$ (the fusion of $t t$ ) or $r r$ (the parts obtained by subdividing $s$ in another way).

Thus, it seems sensible to look at the plural logic relation is one of $(<)$. There are also independent reasons for suspecting that $<$ should be relativized in order to defend strong CAI: it is an immediate source of discernibility between the whole and the parts. Bøhn, who does not discuss the connection between is one of and numerical ascriptions, is nonetheless aware that is one of is intrinsically problematic: 
Assume $a, b$ compose $c$. By CAI, $a, b=c$. [...] $a, b$ has the property of having $b$ as one of them, which $c$ does not. (Bøhn, 2014, p. 147)

$<$ would express a relation that is sensitive to the way in which a portion of reality is conceptualized. The relativization would concern - so to speak - the apparent second argument position of $<$. The idea is that it is not the same thing to be one of the Netherlands, Belgium, and Luxembourg and one of the molecules, in spite of the fact that Netherlands, Belgium, and Luxembourg are identical to the molecules. This is explained by postulating that the expressions "the Netherlands, Belgium, and Luxembourg" and "Benelux" also convey a concept, which in the logical form occupies an additional argument position.

Thus, Belgium is one of the Netherlands, Belgium and Luxembourg from the viewpoint of the concept country. Contra linguistic intuitions, Belgium is also one of Benelux from the viewpoint of the concept country. By contrast, from the viewpoint of the concept multinational entity (as well as from the viewpoint of the concept molecule), Belgium is not one of Benelux, and (contra linguistic intuitions) is not one of the Netherlands, Belgium, and Luxembourg. The impression that these kinds of predication make Benelux discernible from the Netherlands, Belgium, and Luxembourg is thus explained away.

Concerning numerical ascriptions, the paraphrase of (Three Relativized, Unanalyzed) would include the relativization of $<$. Insofar as this relativization consists in the addition of an argument place, we can turn $<$ in a ternary predicate constant $<_{r e l}$, whose first two arguments are the standard ones, while the third argument is the concept (a Polish notation is employed for ternary $\left.\prec_{r e l}\right)$.

$$
\begin{array}{r}
\exists x \exists y \exists z\left(x \neq y \wedge y \neq z \wedge x \neq z \wedge<_{\text {rel }} x t t c \wedge<_{\text {rel }} y t t c \wedge<_{\text {rel }} z t t c \wedge\right. \\
\left.\wedge \forall w\left(<_{\text {rel }} w t t c \rightarrow w=x \vee w=y \vee w=z\right)\right)
\end{array}
$$

(Three with Relativized Is One of)

Here, $t$ can be replaced salva veritate by a singular term for Benelux, or by a plural term for the molecules. From the viewpoint of the concept country, there are three things that are one of Benelux and of the molecules. So far so good.

But we are going to show that the solution comes at a risky price: a highly revisionary stance on plural identity. Let us see why.

Standard plural identity is usually defined as follows:

$$
x x=y y \equiv_{d e f} \forall z(z<x x \leftrightarrow z<y y) \quad \text { (Plural Identity - Definition) }
$$

But $<_{r e l}$ now replaces $<$. How can the definition of plural identity be adapted to $<_{r e l}$ ? The link between plural identity and the relation of being one of is tight, and it would be insane to sever it. Plural identity is a genuine kind of identity insofar as the above, standard definition connects 
it to standard singular, one-one identity. Moreover, the conditions of identity between some things and some other things cannot but mirror the extensional identity conditions between the sets of those things; and two sets are identical iff they have the same elements.

This is probably the reason why the above definition of plural identity is, as far as we know, undisputed in the literature. ${ }^{22}$ If plural identity is to retain its logical status as the plural cousin of standard identity and the expected parallelism with extensional set theory, the relativization of is one of has to be mirrored in some way in the definition of plural identity.

Nonetheless, the combination of (Plural Identity - Definition) with strong CAI is known to bear troublesome consequences, and in particular to lead to the so-called Sider's Collapse, given a seemingly innocent additional assumption, that is the following (Plural Covering). ${ }^{23}$

$$
\forall x \forall y(x P y \rightarrow \exists z z(y=\Sigma(z z) \wedge x<z z)) \quad \text { (Plural Covering) }
$$

It is possible to infer that something is one of some things iff it is part of their fusion.

$$
\forall x x \forall y(y=\Sigma(x x) \rightarrow \forall z(z<x x \leftrightarrow z P y)) \quad \text { (Collapse) }
$$

Given any definition of mereological sum, the left-to-right direction of (Plural Covering) is immediate. In the other direction, suppose $z P y$. Then, by (Plural Covering), there are $w w$ such that $z<w w$ and $y=\Sigma(w w)$. But then $y$ is both the fusion of $x x$ and of $w w$. By strong CAI, $y=x x$ and $y=w w$ and, by the transitivity of identity, $x x=w w$. To exemplify, the Netherlands, Belgium, and Luxembourg would be plurally identical to the molecules in Benelux, and vice versa. Now, given the left-to-right direction of (Plural Identity - Definition), this would mean that whatever is one of the Netherlands, Belgium, and Luxembourg is also one of the molecules in Benelux; this outcome seems unacceptable.

It is worth underlining that, if absolute $<$ is in our language, then the left-to-right direction of (Plural Identity - Definition) - that is used in the above derivation of Collapse - is a mere instance of Leibniz's Law: it is the claim that plural identicals do not differ in what is one of them. But Leibniz's Law is an essential tenet for the variety of strong CAI we are discussing. Thus, strong CAI needs to get rid of absolute $\prec$, by relativizing it, already in order to avoid Collapse, quite independently of the problems about the paraphrases of cardinality ascriptions.

\section{Relativizing Is One of and Plural Identity}

There are two broad ways to mirror the relativization of is one of in (Plural Identity - Definition). The first is to adopt relative plural identity. The second is to keep identity absolute, and adapt the definition of absolute plural identity to the three-places $<_{\text {rel }}$.

\footnotetext{
${ }^{22}$ Bøhn himself refers to (Yi, 2005, 2006) "for the details of plural logic I employ" (Bøhn, 2014, p. 144, n. 3), and in these works Yi defines plural identity through (Plural Identity - Definition) ((Yi, 2005, p. 487); (Yi, 2006, p. 243)), and writes that "is one of is essential to analysing the plural cousin of the identity predicate." (Yi, 2005, p. 488).

${ }^{23}$ (Sider, 2007), (Yi, 1999) and (Sider, 2014). See in particular (Calosi, 2016) and (Calosi, n.d.) for an accurate analysis of the disastrous impact of the Collapse on CAI.
} 
The first broad strategy consists in claiming that plural identity is always relative to a concept. For any concept $c,=_{c}$ will be defined in terms of $\prec_{r e l}$, with $c$ as the concept argument of $<_{\text {rel }}$ in the definiens.

$$
x x={ }_{c} y y \equiv_{d e f} \forall z\left(\prec_{r e l} z x x c \leftrightarrow<_{r e l} z y y c\right) \quad \text { (Relative Plural Identity) }
$$

If plural identity is relative, it is difficult to keep singular identity absolute. After all, $x x$ and $y y$ are usually allowed to take singular values, so that singular identity can be seen as a special case of plural identity. In this special case, definitions such as (Plural Identity - Definition) and (Relative Plural Identity) are not very informative (the only thing that would be one of $x x$ and one of $y y$ would be the self-identical single thing at issue): but also in this case we would be forced to involve a concept $c$.

We have already observed in $\S 5$ that relative identity is an incoherent path for the backers of strong CAI: if you are ready to relativize identity, then you are also ready to admit that indiscernibility does not hold unrestrictedly, and it does not make sense to show that the whole and the parts are absolutely indiscernible.

The second broad strategy consists in reconciling absolute identity and relative is one of. The binary $\prec$ in (Plural Identity - Definition) has to be replaced by the ternary $<_{r e l}$. But what do we do with the additional argument position for concepts? There are three prima facie plausible suboptions:

i) the definition of plural identity could make explicit reference to a specific concept;

ii) the argument position for a concept could be quantified existentially;

iii) the argument position for a concept could be quantified universally.

None of these suboptions is fully satisfactory.

i) would lead to the following redefinition of plural identity:

$$
x x=y y \equiv_{d e f} \forall z\left(<_{r e l} z x x k \leftrightarrow<_{r e l} z y y k\right) \text { (Plural Identity - Relativized to One Concept) }
$$

$k$ is here a constant for a concept. The motivation for this amendment could be that some privileged concept is connected to identity. The privileged concept could be a generic and all-encompassing concept - something like the concept existent, or object, or entity. These concepts could correspond to what Lewis calls "blanket terms". ${ }^{24}$

This amendment presupposes that a generic and all-encompassing concept has been identified. Many candidates for the role could be contested, but this is probably not an insuperable obstacle. Meinongians could insist that plural identity can also be applied to non-existent objects,

\footnotetext{
${ }^{24}$ See for example (Lewis, 1986, p. 99). A theory of blanket terms - under the sobriquet of transcendentals - is outlined in (Lando and Spolaore, 2014).
} 
and that the concept existent is, as a consequence, unfit for the role of $k$ in (Plural Identity Relativized to One Concept); the concept object would be preferable. But other philosophers could observe that "object" is often used in a more selective way (e.g., to designate only so-called ordinary objects). ${ }^{25}$ Perhaps the concept entity could make everyone happy. Otherwise, the generic, all-encompassing concept could be designated by convention.

Suppose, in any case, that we settle on a certain concept in the role of $k$, say the concept entity $\left(c_{e}\right)$. Such a concept would allow us to redefine the old two-place $<$, under the guise of $\prec_{a b s}$. We could say that $x$ is absolutely one of $y y$ iff $x$ is one of $y y$ from the viewpoint of $c_{e}$ :

$$
x \prec_{a b s} y y \equiv_{d e f}<_{r e l} x y y c_{e}
$$

But the introduction in our language of an absolute kind of $<$ leads to Collapse, by the simple application of Leibniz's Law we mentioned at the end of $\S 6$ : if $x x=y y$, then whatever is absolutely one of $x x$ is absolutely one of $y y$ and vice versa.

The following redefinition of plural identity would ensue from (ii), instead:

$$
x x=y y \equiv_{d e f} \exists c \forall z\left(<_{\text {rel }} z x x c \leftrightarrow<_{\text {rel }} z y y c\right) \quad \text { (Plural Identity - Relativized Existentially) }
$$

The requirement would be that there is some concept or other, from the viewpoint of which something is one of $x x$ iff it is one of $y y$.

But compare the Gina portion of reality (Gina is the sum of the two pets in our first example) with the portion of reality that comprises all of Gina and some air in the middle of Mina's coat. Both portions of reality are composed of molecules. Let $u u$ be a term for the molecules in Gina, and $v v$ a term for the molecules in Gina + the air in the middle of Mina's coat. Now, from the viewpoint of the concept pet, it seems that Mina, Gino, and nothing else are one of $u u$, and that Mina, Gino, and nothing else are one of $v v$. This means that there is a concept (namely, the concept pet) that satisfies (Plural Identity - Relativized Existentially). As a result, $u u=v v$ : the molecules in Gina would be identical to the molecules in Gina + the air in Mina's coat. But this is clearly wrong. (Plural Identity - Relativized Existentially) is a bad way to amend (Plural Identity - Definition), and we can drop it. ${ }^{26}$

The last possible option is iii), which would redefine plural identity as follows:

\footnotetext{
${ }^{25}$ See for example (Korman, 2015, pp. 39-40).

${ }^{26}$ One could argue that $<_{\text {rel }} x$ yy $c$ should require that the concept $c$ allows for an exhaustive subdivision of the portion of reality corresponding to $y y$. However, this would mean defining $<_{r e l}$ as follows, and thus in terms of: a) an absolute kind of is one of; b) mereological fusion.$$
\left.\prec_{\text {rel }} x \text { yy } c \equiv_{\text {def }} \exists z z(\forall w(w<z z \rightarrow w \text { falls under } c)) \wedge \Sigma(z z)=\Sigma(y y)\right)
$$

a) would lead us to introduce absolute $<$ in our language, and thus to Collapse. Also b) could be deemed undesirable, insofar as a logical notion should not be defined in terms of mereological ones (but - as we already conceded in $\S 3-$ a backer of strong CAI could insist that mereology is logic).
} 
$x x=y y \equiv_{d e f} \forall c \forall z\left(<_{r e l} z x x c \leftrightarrow<_{r e l} z y y c\right) \quad$ (Plural Identity - Relativized Universally)

In this case, the idea would be that plural identicals $x x$ and $y y$ are required to be such that, irrespectively of the $c$ from the viewpoint of which they are conceptualized, any single thing $z$ is one of $x x$ under the viewpoint of $c$ iff $z$ is one of $y y$ under the viewpoint of $c$.

Thus, the definition of plural identity would involve every way of conceptualizing the plural identicals. The definiens in (Plural Identity - Relativized Universally) is an uncontroversial necessary condition for identity. Indeed, the left-to-right direction of (Plural Identity - Relativized Universally) is nothing other than an instance of Leibniz's Law: if $x x$ and $y y$ were such that something is one of $x x$ but not of $y y$ (or vice versa) from a certain viewpoint (where the viewpoint is nothing more than a relatum of $<_{r e l}$ ), then $x x$ and $y y$ would be discernible.

In iii) the condition is not merely necessary: (Plural Identity - Relativized Universally) is a definition of plural identity, and we would be forced to involve in the definition of plural identity every concept, including highly specific concepts such as square, square of $4 \mathrm{in}^{2}$, pet, and country. The price of thus defining absolute identity in terms of relative is one of is that plural identity loses its purely formal status, and becomes dependent on highly specific and far from topic-neutral concepts. ${ }^{27}$

One could note that something similar happens in Leibniz's Law itself, and that nobody thinks that Leibniz's Law makes identity a non-formal notion. Indeed Leibniz's Law, in its second-order form, quantifies over every property:

$$
\forall x \forall y(x=y \rightarrow \forall F(F x \leftrightarrow F y))
$$

(Leibniz's Law)

However, there are two relevant differences between the case of Leibniz's Law and (Plural Identity - Relativized Universally).

a) Leibniz's Law is not an explicit definition of identity. While it is a pivotal part of the standard characterization of identity, it is not expected to provide a full characterization of what identity is. Leibniz's Law yields only a necessary - and not sufficient - condition for identity. Of course, together with reflexivity, Leibniz's Law provides a so-called implicit definition of identity. Nonetheless, Leibniz's Law is not expected to explain what being identical consists in, in terms of a totality of properties.

b) The quantification over all properties in Leibniz's Law is really an indiscriminate quantification over every feature, no matter how gerrymandered, extrinsic, relational, or trivial. This is the reason why there is also a schematic version of Leibniz's Law, in which you can put any formula of your language and obtain an instance of the schema. The total lack of discrimination among the properties at stake in Leibniz's Law preserves its

\footnotetext{
${ }^{27} \mathrm{~A}$ similar issue also affects i) (and is quite independent of the other problems of i) discussed above), but is more serious in the case of iii). Indeed, in the case of i), identity would be defined in terms of a generic, all-encompassing concept, that could qualify as formal in itself, in a sense inspired by Husserl's or Wittgenstein's formal concepts. By contrast, in the case of iii), identity would be defined in terms of clearly non-formal concepts.
} 
topic-neutrality. Indeed you do not need to delimit any specific domain of features for which the law holds. By contrast, in the case of (Plural Identity - Relativized Universally), in spite of the fact that you quantify over every concept, the totality of concepts is still a controversially delimited domain of viewpoints from which it makes sense to divide up a plurality. Should we admit only sortals, and, if so, what is a sortal? Do relational concepts belong to the totality of concepts that are here at stake? When we ask whether some stars are identical to some others, should we care about how they are divided up from the viewpoint of the concept car, or of the concept half car, or of the concept being one mile from Berlin, Germany? Perhaps there are sensible answers to these questions, but they are unlikely to be free from controversial metaphysical and semantical assumptions, from which the definition of plural identity should stay clear, if identity is to retain its status of formal, topic-neutral notion. ${ }^{28}$

We are not going to argue in this paper in favour of the default thesis that identity (including plural identity, which is tightly connected with standard one-one identity) is a topic-neutral, formal, or logical notion. But there is clearly a significant philosophical cost in deviating from this default thesis. If a backer of CAI opts for this route, it is up to her to show that this cost is worth paying.

This completes our analysis of the ways in which the backers of strong CAI can relativize numerical ascriptions, while insisting - contra Frege - that objects are their proper subjects. Given that numerical ascriptions can be paraphrased in plural logic, the relativization of numerical ascriptions requires the relativization of something else in the paraphrase. But these relativizations either do not work, or lead to Collapse, or imply high philosophical costs.

\section{Counting Concepts?}

In the previous sections we have shown that there is no easy way to be partially Fregean about numerical ascriptions. If we relativize numerical ascriptions while insisting - contra Frege - that their subjects are objects, we come up against various problems and controversial philosophical commitments with the usual paraphrases of numerical ascriptions in terms of quantifiers, identity, and being one of. Consider, again, the NDA argument:

(P1) $t$ is the sum of $u u$;

(P2) $u u$ are three and not one; $t$ is one and not three;

(C) It is not the case that $t$ and $u u$ are identicals.

Up to now in our analysis, it has been difficult to reject the premiss P2 of NDA and to thereby block it. The second broad option for strong CAI is to be more faithful to Frege and hold that objects are not the proper subjects of numerical ascriptions. The linguistic datum that Benelux can be said to be one (multi-national entity), to be three (countries), or to be a very

\footnotetext{
${ }^{28}$ About the problem of delimiting the domain of concepts at stake in cardinality ascriptions, see again (Koslicki, 1997).
} 
large number (of molecules) should not lead us to analyze these ascriptions about Benelux in order to make them mutually compatible. Instead, we should take these incompatibilities as a sign that numerical ascriptions are not about objects.

According to Frege, the subjects of these statements are certain kinds of concepts, and the predicates "to be one", "to be three", and "to be a very large number" are more appropriately expressed by locutions such as "to have one instance", "to have three instances", and "to have a very large number of instances" of a certain concept. For Frege, concepts do not occupy additional argument places for numerical predicates (as Bøhn thinks), but are the only proper subjects of cardinality ascriptions. In Frege's words, "the content of a statement of number is an assertion about a concept" (Frege, 1950, §46).

In $\S \S 10-12$ we will examine the elaborate way in which Aaron Cotnoir, in his theory of general identity set forth in (Cotnoir, 2013), attempts to follow this strategy for the defence of CAI. Cotnoir does this indirectly, namely by replacing concepts with a certain kind of sets in the role of real subjects of numerical ascriptions. We will show that Cotnoir's strategy is partially successful in terms of numerical ascriptions and indiscernibility, but is affected by other problems and difficulties.

Is there a more direct way of following the second broad strategy for rejecting P2? So could strong CAI go fully Fregean instead, and directly hold that concepts are their real subjects? No. The problem is that this option would not really help the cause of strong CAI. This is probably why, as far as we know, no backer of CAI has chosen this simple route. Nonetheless, it is interesting to dwell on why strict Fregean counting is not apt for this role, and this will also help us to appreciate the peculiarities of Cotnoir's alternative.

The problem is that the role of concepts is to distinguish between different ways of partitioning a portion of reality. As a consequence, the concepts themselves (the proper subjects of cardinality ascriptions, according to Frege) end up providing a counterexample to the alleged indiscernibility of the objects falling under them.

Concepts have the primary function of specifying and isolating what falls under them, and what falls under the two concepts in the example is/are not the same thing(s). In Frege's famous example of the Iliad, there is (are) not some thing(s) that fall(s) both under the concept being $a$ book of the Iliad and under the concept being the Iliad poem, in spite of the fact that the poem consists of (twenty-four) books.

The unanalyzed objects or phenomena that are discussed in the pars destruens of Frege's analysis of cardinality ascriptions (which may correspond to what the contemporary backers of strong CAI call "portions of reality") have simply no role in his positive view. The books of the Iliad and the Iliad poem fall under different concepts, which make them discernible and numerically different.

In their references to Frege's Foundations of Arithmetic, the backers of CAI tend to quote excerpts from the pars destruens, in which the unanalyzed phenomenon or portion of reality is discussed (and finally rejected) as a candidate subject for numerical ascriptions. This already quoted passage refers to an "external phenomenon":

While looking at one and the same external phenomenon, I can say with equal truth both "It is a copse" and "It is five trees", or both "Here are four companies" and "Here are 500 men". (Frege, 1950, §84) 
But this "external phenomenon" is not something that is preserved as such in Frege's positive ontology: it is neither an object falling under some concepts, nor a concept. In Frege's positive account of the example involving a copse and the trees in it, there are two concepts with a different number of instances, and then different objects in their extensions. The trees make up the copse, but they are not in any sense identical to it. Frege was not a forerunner of strong CAI, and not only because the theory of composition was not his primary concern.

\section{Counting Sets?}

Given that concepts do not help, let us see how Fregean, second-order counting can be developed set-theoretically. There is a well-known affinity between concepts and sets. According to Frege, any concept has an extension. This extension includes all the objects that the concept (which, according to Frege, is a function) maps to the truth value true. In terms of cardinality, a concept $c$ falls under the second-order concept having exactly $n$ instances iff its extension includes exactly $n$ instances of $c$.

In the Foundations of Arithmetic Frege refrains from identifying extensions with sets or collections (and denies that sets or collections are the proper subjects of cardinality ascriptions), because he saw these latter notions as being worryingly vague. However, this stance was about pre-Cantorian notions of sets and collections, and later in his career Frege conceded that extensions are actually classes. ${ }^{29}$

After the discovery of set-theoretic paradoxes, it is - to say the least - contentious to claim that for every concept there is the set of things instantiating it. Nonetheless, also once the domain of sets has been appropriately restricted by an axiomatic set theory, we have sets for a huge amount of concepts. In particular, when we count parts of concrete objects (such as books that divide up poems), there is no great difference between attributing to the concept being $a$ book of the Iliad a certain number of instances, and attributing a certain cardinality to the set of objects falling under that concept.

In answering a letter from Husserl, according to whom extensions - and not concepts were the most fundamental bearers of cardinality, Frege claims that there is no relevant priority or fundamentality at stake:

I will not quibble over whether a statement is directly about the concept and indirectly about its extension, or indirectly about the concept and directly about its extension, for one goes with the other. (Frege, 1984, p. 322)

As a consequence, the set-theoretic approaches to cardinality are generally and rightly considered Fregean, although they do not involve concepts at all.

The difference is in fact so little that, by itself, the replacement of concepts with sets is not enough to help the backers of strong CAI. As in the case of concepts, numerical ascriptions are in a sense set aside as counterexamples to the indiscernibility of the whole and the parts, simply because the whole and the parts are not the real subjects of numerical ascriptions; the

\footnotetext{
${ }^{29}$ For example in a letter to Russell on July 28th 1902 (Frege, 1980, pp. 139-142). See also (Blanchette, 1999, n. 7) for discussion.
} 
real subjects are sets. But, as in the case of concepts, sets give us new counterexamples to the indiscernibility of their elements.

Consider $\{$ Benelux $\}$, i.e. the singleton of Benelux. Only the whole - Benelux - is an element of $\{$ Benelux $\}$, while the Netherlands, Belgium, and Luxembourg are not individually elements of $\{$ Benelux $\}$. One could try to propose that the Netherlands, Belgium, and Luxembourg are collectively members of $\{$ Benelux $\}$, but set-theoretic membership seems to be a canonical case of one-one relation between a set or an individual on one side and a set on the other: it is not clear how it could be construed as a many-one, collective relation.

In order to take a step forward we need to choose the right sets: the mere extensions of concepts do not work. In the next three sections we will interpret (Cotnoir, 2013)'s theory of general identity as an attempt in this direction, which is somewhat successful in extending indiscernibility to composition, but highly problematic from other points of view.

\section{Counting Subsets of Atoms in a Portion of Reality: Cotnoir's General Identity}

In his theory of general identity (GI) Cotnoir introduces a sort of set-theoretic bridge between the whole and the parts: the set of the atoms in a certain portion of reality.

This bridge between the whole and the parts was missing in the second-order approaches to numerical ascriptions discussed in the two previous sections. We have seen that both in the case of concepts (§8) and in that of extensions/sets ( 99$)$, the problem is that they end up introducing new counterexamples to indiscernibility for composition. These counterexamples are generated by the introduction of the real subjects of numerical ascriptions: concepts and sets are introduced as real bearers of numerical ascriptions, and then parts and whole diverge in their relations to these real bearers (the relation of falling under in the case of concepts; set-theoretic membership in the case of sets).

We need to find some kind of role for the portion of reality in our theory of numerical ascriptions, in order to prevent them from being endowed with totally disconnected subjects, which end up being a source of discernibility.

In Cotnoir's GI, the role of a portion of reality is played by the set of its mereological atoms, that is - in the case of Benelux - the set of those parts of Benelux that have no proper part. For any portion of reality, there is the set of mereological atoms in it. The atoms can be exhaustively grouped in different ways, thereby obtaining various sets of sets of atoms. These sets of sets of atoms are partitions (if the subsets of atoms are mutually disjoint) or covers (if the subsets are allowed to overlap) of the set of atoms. ${ }^{30}$

The intuitive expectation is that a new notion of identity would connect anything that is the same portion of reality. Since atoms play the role of the portion of reality, GI will connect anything that is made, ultimately, of the same atoms. Benelux will be generally identical to the

\footnotetext{
${ }^{30}$ The distinction between partitions and covers is pivotal in Cotnoir's treatment of collective and cumulative predications. It is not equally important in the case of numerical ascriptions, and in what follows we skip over it in most cases for the sake of simplicity. Most of our references to a way of subdividing or partitioning or divvying up the atoms can be taken indifferently for a partition or a cover. The only exception is at the end of $\S 12$, where it matters how many subsets of the set of atoms are countenanced.
} 
Netherlands, Belgium, and Luxembourg, and will also be generally identical to the molecules in that portion of reality. The Netherlands, Belgium, and Luxembourg will also be generally identical to these molecules.

The general binary identity predicate $\approx$ admits singular and plural terms in both argument positions. Cotnoir provides truth conditions for GI statements of the four resulting forms (many-many, many-one, one-many, one-one). In the following equivalences, $U$ stands for set-theoretic union, and, for any expression $x, \bar{x}$ stands for the denotation of $x$.

$$
\begin{aligned}
& x x \approx y y \text { is true iff } \cup \overline{x x}=\bigcup \overline{y y} \text { (many-many) } \\
& x x \approx y \text { is true iff } \bigcup \overline{x x}=\bar{y} \text { (many-one) } \\
& x \approx y y \text { is true iff } \bar{x}=\bigcup \overline{y y} \text { (one-many) } \\
& x \approx y \text { is true iff } \bar{x}=\bar{y} \text { (one-one) }
\end{aligned}
$$

The denotation of a singular term is a set of atoms, while a plural term denotes a set of these sets. "Benelux" denotes a set of atoms. The plural term "the Netherlands, Belgium, and Luxembourg" denotes a set of sets of atoms. The one-many GI statement connecting these two terms is true, because the atoms involved are the same: making the set-theoretical union of the set of the three sets of atoms (corresponding to the three countries) we obtain the same set of atoms which is denoted by "Benelux". The many-many GI statement connecting "the Netherlands, Belgium, and Luxembourg" and "the molecules in Benelux" is also true.

Cotnoir's strategy is meant to show that indiscernibility unrestrictedly holds for GI. He also discusses in depth the problems raised by various kinds of collective predications, but here we are interested only in the case of numerical ascriptions. Prima facie the countries in Benelux are three, while the multinational entity is one (and not three), and the molecules are a very large number (and neither three nor one). These apparent forms of discernibility of general identicals are dispelled by Cotnoir by requiring that the evaluation of the involved sentences takes account of how the domain of atoms in the portion of reality is or can be subdivided.

This can happen in two ways. Either in an indexical form, so that "The molecules are three" is false because there is a contextual parameter that makes the evaluation relative to the division of Benelux in molecules, while "The Netherlands, Belgium, and Luxembourg are three" is true because there is a contextual parameter that makes the evaluation relative to the division of Benelux in countries. Or in a sub-valuational form, where it is enough for the truth that there is a division of Benelux such that its pieces are three, so that both "The molecules are three" and "The Netherlands, Belgium, and Luxembourg are three" come out true. In both cases, Leibniz's Law holds. In the indexical variant, the alleged exception to indiscernibility proves to be a misleading appearance, and fades once we consider the appropriate contextual parameter. In the sub-valuational variant, the terms of true GI statements can actually be substituted salva veritate, so that the alleged exception is instead a confirmation of Leibniz's Law.

In Cotnoir's proposal, what are counted are the elements of a set of sets of atoms. The indexical and the sub-valuational variants differ in how they pinpoint the source of the counting, i.e. that division (partition or cover) of the domain of atoms in various subsets that really matters for numerical ascriptions: the set of these subsets of atoms is what is actually counted. In the indexical version, a contextual parameter tells us to count a certain kind of subsets of atoms, those that are the elements of the set of sets of atoms denoted by the subject term. The plural 
expression "the Netherlands, Belgium, and Luxembourg" introduces a contextual parameter that selects the country-like sets of atoms as being those that should be actually counted. The referent of the plural expression is the set of these sets. In the sub-valuational variant, the exact referent of the subject expression does not really matter. It is enough that the domain of atoms in the portion of reality can be divvied up in such a way that the set of sets of atoms has the cardinality indicated by the numerical ascription.

It is worth noting that in Cotnoir's framework there is no need to relativize identity, quantification, or set-theoretical notions, in order to paraphrase numerical ascriptions in logical terms. In other words Cotnoir avoids the conundrum faced by the first-order approaches discussed in $\S \S 2-6$, in which something should be relativized, but nothing is such that it is desirable for strong CAI to relativize it.

What is relativized (and only in the indexical variant) is the set that matters for the evaluation of the ascription. Once the set is chosen, counting is absolute and can be expressed through standard, unrelativized quantifiers, negation, membership, and one-one identity, without any need to involve GI. To say that the set of the country-sets of atoms in Benelux (let $C$ be this set of sets of atoms) has three elements is tantamount to making the following formal claim:

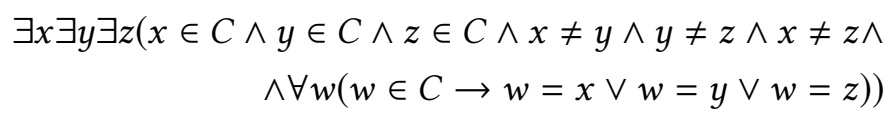

\section{The Failure of Coreferentiality}

Cotnoir's GI could be criticized from several points of view. For example, atoms play a pivotal role in GI, and it could be contentious to assume that in any portion of reality there is a domain of atoms. Does this mean that GI cannot be applied as such to any case of so-called gunk, that is to entities such that all their proper parts always have further proper parts?

In (Carrara and Lando, 2016) we raised a different objection against GI. We argued that Cotnoir's set-theoretical approach violates the following semantic requirement on genuine identity statements:

Coreferentiality Constraint The terms of a true identity statement must be coreferential.

How does Cotnoir's GI end up violating the Coreferentiality Constraint? Let us look at the identity conditions for GI statements laid down in $\S 10$. In each of the three cases in which a plural term is involved, the sets denoted by the terms of a true identity statement are allowed to be different sets. They differ from the viewpoint of standard, uncontroversial identity conditions for sets, according to which two sets are identical iff they have the same elements. In the two cases (many-one, one-many) where one term is singular and the other plural, one term denotes a set of atoms and the other denotes a set of sets of atoms. In the many-many case the two terms are allowed to denote two different sets of sets of atoms. In the Benelux example, the set 
of the country-sets of atoms is not the set of the molecule-sets of atoms, and neither of them is the Benelux-set of atoms.

Once the Coreferentiality Constraint is disobeyed, indiscernibility does not show that GI is a genuine identity relation. ${ }^{31}$ When standard identity is at stake, the principle of indiscernibility is seen as non-negotiable insofar as it boils down to the hardly controversial claim that anything has just the properties it has. However, the principle does not say exactly this: it says that anything has just the properties that anything identical to it has. This can be turned into the platitude that anything has the properties which it itself has only if identity is meant, as usual, as that relation that anything has with itself and with nothing else. However, Cotnoir's GI is not such a relation. What are the relata of the GI relation expressed by $\approx$ ? Well, as in any other statement with a relational predicate, the relata of the relation are the denotations of the terms that are the arguments of the relational predicate. Thus, in the many-many instance of the Benelux example, we have the set of the country-sets of atoms on the one hand, and the set of the molecule-sets of atoms on the other. They are different sets, with different elements.

As we know, according to Sider, a defender of strong CAI who denies Leibniz's Law "would arouse the suspicion that their use of 'is identical to' does not really express identity". (Sider, 2007, p. 59) But this same suspicion is aroused by the claim that a sentence obtained filling the gaps in "... is (generally) identical to ..." with referential expressions can be true even if the referents of these expressions are distinct, and is aroused exactly in the struggle to impede that suspicion from being aroused by Leibniz's Law.

The problem here is that, once it has been conceded that the denotations of the arguments of $\approx$ are different, no evidence can be provided in favour of the thesis that the relation expressed by $\approx$ is an identity relation.

In (Carrara and Lando, 2016) we spelt out many details of Cotnoir's violation of the Coreferentiality Constraint, and showed that GI could be amended, and thus made compliant with it, if the terms of GI statements were hyperplurals, instead of denoting sets. ${ }^{32}$ The idea is that both "the Netherlands, Belgium, and Luxembourg" and "Benelux" would denote plurally the mereological atoms in Benelux, but at different levels of plurality; also "the molecules" would denote those same atoms at the same level of "the Netherlands, Belgium, and Luxembourg", but would group (in a non-very clear sense) them differently.

The resort to hyperplurals is highly controversial in itself. ${ }^{33}$ But it should be conceded that hyperplurals would solve Cotnoir's problem with the Coreferentiality Constraint. However, we are going to see in the next section that Cotnoir's treatment of cardinality ascriptions, in particular when it is detached from its set-theoretical character, raises other concerns.

\section{General Identity and Numerical Ascriptions}

Consider an instance of our NDA argument which concerns Benelux. The opponents of CAI say that the Netherlands, Belgium, and Luxembourg are three, while the molecules are a very large

\footnotetext{
${ }^{31}$ For a different criticism to CAI based on the coreferentiality constraint see (Calosi, n.d.) on the Semantic Failure.

${ }^{32}$ (Cotnoir, 2013) himself points briefly (p. 301) to the possibility of framing general identity in term of hyperplurals, and to (Rayo, 2006) for a suitable implementation.

${ }^{33}$ See (McKay, 2006, pp. 46-53) and (Uzquiano, 2004) for various perplexities.
} 
number, and are not three; thus, the Netherlands, Belgium, and Luxembourg are discernible from the molecules (and, as a consequence, not identical to them).

Cotnoir, in his indexical mood, replies as follows: yes, but this is no exception to indiscernibility, because in evaluating "the Netherlands, Belgium, and Luxembourg are three" and "the molecules are three" we should take into account a hidden indexical, which makes the first sentence true and the latter false. This indexical element individuates the proper source of counting in a way of slicing the domain of atoms, that is - in the official, set-theoretical variant of GI (the only one that Cotnoir develops) - of grouping it in a set of sets of these atoms.

Cotnoir, in his sub-valuational mood, instead replies as follows: there is no exception to indiscernibility; both the Netherlands, Belgium, and Luxembourg on the one hand, and the molecules on the other are three (and a very large number as well). In fact, the union of the set of sets of atoms denoted by "the Netherlands, Belgium, and Luxembourg" is also the union of the set of sets of atoms denoted by "the molecules". And this union can be subdivided in such a way that we obtain a three-membered set of sets of atoms.

In this set-theoretical framework, the two subject terms of "the Netherlands, Belgium, and Luxembourg are three" and "The molecules are three" (in spite of also being the terms of a true GI statement) are not coreferential. By contrast, in the hyperpluralist version of GI we sketched in (Carrara and Lando, 2016), the two terms are coreferential.

But, in the indexical variant, the non-coreferentiality of terms in true GI statements could be suspected to be key in treating numerical ascriptions and extending indiscernibility to composition. In fact, it is not clear in Cotnoir's theory what determines or suggests the indexical parameter selecting a certain set (and not another) as the set to be actually counted in evaluating a cardinality ascription. A plausible way to fill this gap is to say that the set to be counted is the one denoted by the referential expression that flanks the numerical predicate. Thus, the sentence "the word "molecule" would suggest that the sentence "the molecules are three" should be evaluated according to a certain indexical parameter; this indexical parameter selects the set of sets of atoms that includes a set of atoms for each molecule in Benelux.

One and the same feature of GI - namely, the fact that a true GI statement is allowed to include non coreferential terms - would violate a pivotal constraint on genuine identity relations and grant the extension of indiscernibility. Composition would respect Leibniz's Law, but only at the price of violating the Coreferentiality Constraint: no overall progress for the idea that a whole is genuinely identical to its parts.

Instead, what could happen if the referential expressions in cardinality ascriptions are hyperplurals? Precisely because the Coreferentiality Constraint is respected, the referents of "the molecules" and of "Benelux" cannot be of help in selecting different sets. One could perhaps make appeal to a non-referential component of the semantics of hyperplurals, but - at least in the present state of the literature on hyperplurals - it is unclear what this component could consist in.

By contrast, in the sub-valuational variant, the evaluation of numerical ascriptions is arguably disconnected from the referents of their subjects. If we opt for covers over partitions, a numerical ascription is true on the necessary and sufficient condition that the number attributed is not greater than the cardinality of the power set of the set of atoms in the portion of reality. If we opt for partitions over covers, the number attributed should not be greater than the 
cardinality of the set of atoms itself.

This leads to the following surprising and apparently unacceptable consequences.

a) The expected numerical incompatibilities do not hold. For example, both the molecules and the countries are both three and a very large number. In fact, the set of atoms in that portion of reality can be partitioned or covered by a three-membered or by a many-membered set of sets of atoms. ${ }^{34}$

b) Lots of seemingly false numerical ascriptions are licensed as true; say that there are $n$ atoms in Benelux; for any $m \leq 2^{n}$ (in the case of covers), or for any $m \leq n$ (in the case of partitions), "The Netherlands, Belgium, and Luxembourg are $m$ " is true. The ensuing semantic revisionism is hard to overestimate.

c) Plausibly enough (Cotnoir does not discuss this point), the semantics of comparative operators such "are as many as" is tied to that of numerical ascriptions, in the following way: for any $x x$ and any $y y$, " $x x$ are as many as $y y$ " is true iff there is a number $n$ such that both " $x x$ are $n$ " and " $y y$ are $n$ " are true. If so, "The Netherlands, Belgium, and Luxembourg are as many as the molecules composing them" would turn out to be surprisingly true. ${ }^{35}$

d) The interaction between is one of and cardinality ascriptions turns out to be deviant. Belgium is one of the Netherlands, Belgium, and Luxembourg. But it may happen that there is a number $m$ and there is a number $n$ such that $m<n$, while "Belgium is $n$ " is true and "The Netherlands, Belgium, and Luxembourg are $m$ " is true as well.

e) The semantic evaluation of numerical ascriptions would seem to require, epistemically, both an awareness of the number of atoms in a portion of reality, and, in the case of covers, an ability to calculate the cardinality of the power set of the set of these atoms. ${ }^{36}$

To sum up, the indexical variant seems to be tied with the non-coreferentiality of terms in several true GI statements. By contrast, the sub-valuational variant does not really exploit the violation of the Coreferentiality Constraint, but leads to the hardly acceptable results a)-e). In both cases, numerical ascriptions are a challenging constraint on how Cotnoir's GI could be developed in a cogent manner.

\footnotetext{
${ }^{34}$ This anomaly could be attributed to the well-known logical peculiarities of sub-valuational (and supervaluational) approaches, and, as usual in these cases, the expected incompatibilities are preserved within the perimeter of each single evaluation. Nonetheless, the anomaly seems to be strictly related to the other side effects b), c), and d).

${ }^{35}$ An alternative way to characterize the tie would be to say that for any $x x$ and any $y y$, " $x x$ are as many as $y y$ " is true iff for every number $n$ " $x x$ are $n$ " is true iff " $y y$ are $n$ " is true. However, the outcome does not change: "Netherlands, Belgium, and Luxembourg are as many as the molecules composing them" would be true.

${ }^{36}$ The degree of unacceptability of e) seems to depend on the extent at which Cotnoir's proposal is meant to provide a semantics for natural language, or merely for a specialized formal language. This is not made clear in (Cotnoir, 2013).
} 


\section{Conclusion}

There are different ways of relativizing numerical ascriptions. Only a few of them promise to be of help in countering the claim that, when $x$ have some parts $y$ and $z, x$ is one and not two, while $y$ and $z$ are two and not one; and in thereby blocking the NDA argument.

If objects are thought to be the proper subjects of numerical ascriptions, the only advisable strategy is to relativize the plural logical relation of is one of. But either we end up embracing relative identity (contra strong CAI's insistence on conservativity about identity and Leibniz's Law), or Sider's Collapse ensues, or identity is entangled with a difficult theory of concepts, and risks being deprived of its expected formal status.

The only promising alternative involves sets of atoms and sets of sets of atoms in portions of reality. These sets are what is properly counted, but are also what is properly identical. The resulting notion of general identity violates, in Cotnoir's set-theoretical formulation, the Coreferentiality Constraint. As a consequence, general identity would not deserve to be considered an identity relation at all. And, also if general identity might be modified to respect the Coreferentiality Constraint, its way of treating cardinality ascriptions relies on controversial and underexplored semantic theories, and leads in some cases to a disappointing semantic analysis.

Lewis's objection against the claim that composition works exactly like one-one identity as summarized in NDA - is still powerful: it is hard to deny that the whole is one, and the parts are many. ${ }^{37}$

It is worth here repeating that nothing in our analysis runs counter to other varieties of CAI. Neither Baxter nor Lewis were completely conservative about identity. This is very clear in the case of Baxter, who rejects Leibniz's Law. But also Lewis legitimizes composition as an identity-like relation that does not really work like one-one identity, and as a result seems to incline towards a kind of pluralism about identity: composition is an identity relation, but is not one-one identity, thus there is more than one identity relation. ${ }^{38}$ In our analysis of cardinality ascriptions, CAI actually squares better with revisionist, non-Quinean views of identity: perhaps a form of relative identity, perhaps a milder kind of pluralism about identity.

What leads to trouble or requires controversial assumptions is the pretension to endorse CAI while being perfectly Quinean about identity: for example, (Bøhn, 2014) claims in the very first page of his essay that "identity is the primitive relation everything bears to itself and to nothing else, uniquely characterized" by reflexivity and Leibniz's Law (p. 143). CAI is then presented as a mere broadening of the domain of identity: the token relations between wholes and their parts and between different slicings of a same whole would be simply new instances of the same old relation. By contrast, Baxter and Lewis admit that the relation at stake is not standard, Quinean, logical identity. And both accept the platitude that Mina and Gino are two, while Gina is one. As shown in this paper, the path to reject this platitude is narrow and perilous.*

\footnotetext{
${ }^{37}$ Perhaps it is possible to interpret cardinality ascriptions in a radically non-Fregean way, without involving concepts. The only attempt in this direction has been outlined in (Wallace, 2011b). See n. 5 above.

${ }^{38}$ See n. 8 above.

*Some preliminary versions of this paper have been presented at the Realism and Objectivity conference in Matera (September 2015), at the Sixth Meeting of the Portoguese Society for Analytic Philosophy in Ponta Delgada
} 


\section{REFERENCES}

Alston, W. and J. Bennett (1984), "Identity and Cardinality: Geach and Frege”, The Philosophical Review, 93, 4, pp. 553-567.

BAXter, D. (1988a), "Identity in the Loose and Popular Sense", Mind, 97, pp. 575-582.

BaXter, D. (1988b), "Many-One Identity", Philosophical Papers, 17, pp. 193-216.

Blanchette, P. (1999), "Relative Identity and Cardinality", Canadian fournal of Philosophy, 29, 2, pp. 205-223.

BøHn, E. (2009), Composition as Identity: A Study in Ontology and Philosophical Logic, $\mathrm{PhD}$ thesis, Amherst: University of Massachusetts.

Bøнn, E. (2011), “Commentary on David Lewis, Parts of Classes”, Humana.Mente, 19, pp. 151-158.

Bøнn, E. (2014), "Unrestricted Composition As Identity", in Composition As Identity, ed. by

D. BAXter and A. Cotnoir, Oxford: Oxford University Press, pp. 143-165.

Bricker, P. (2015), "Composition as a Kind of Identity", Inquiry, Online First, pp. 1-31.

Calosi, C. (2016), "Composition Is Identity and Mereological Nihilism”, The Philosophical Quarterly, 263, 1, pp. 219-235.

CAlosi, C. (n.d.), "Failure or Boredom. The Pendulum of Composition as Identity", American Philosophical Quarterly, Forthcoming.

Carrara, M. and G. LANdo (2016), "Composition, Indiscernibility, Coreferentiality”, Erkenntnis, 81, 1, pp. 119-142.

Carrara, M. and E. Sacchi (2007), "Cardinality and Identity", fournal of Philosophical Logic, 36, 5, pp. 539-556.

Cotnorr, A. (2013), “Composition as General Identity”, in Oxford Studies in Metaphysics, vol. 8, ed. by K. Bennett and D. Zimmermann, Oxford: OUP, pp. 295-322.

Frege, G. (1950), The Foundations of Arithmetic, translated by J. Austin, New York: Harper \& Brothers.

Frege, G. (1980), Philosophical and Mathematical Correspondence, Chicago: University of Chicago Press.

Frege, G. (1984), Collected Papers on Mathematics, Logic, and Philosophy, Oxford: Blackwell.

GeACH, P. (1967), "Identity", Review of Metaphysics, 21, 1, pp. 3-12.

Hovda, P. (2005), "Two Defenses of Composition as Identity", Unpublished manuscript.

KIng, J. (2003), "Tense, Modality, and Semantic Values", Philosophical Perspectives, 17, 1, pp. 195246.

Konman, D. (2015), Objects: Nothing Out of the Ordinary, Oxford: OUP.

Koslicki, K. (1997), "Isolation and Non-Arbitrary Division", Synthese, 112, 3, pp. 403-430.

LANDo, G. (2017), Mereology. A Philosophical Introduction, London: Bloomsbury.

Lando, G. and G. Spolaore (2014), "Transcendental Disagreement", The Monist, 97, 4, pp. 597620.

LEwis, D. (1986), On the Plurality of Worlds, Oxford: Blackwell.

(September 2015), and at the Eidos metaphysics seminar in Geneva (February 2016). We would like to thank Claudio Calosi, Pasquale Frascolla, Ghislain Guigon, Robert Michels, Kevin Mulligan, Matteo Plebani, Pierre Saint-Germier, Maria Scarpati, Alexander Skyles, Timothy Williamson, and all the other attendees for their insightful questions and objections. We would also like to thank Claudio Calosi, Simone Gozzano, Massimo Mugnai, and two anonymous referees for their useful comments on the manuscript. 
Lewis, D. (1991), Parts of Classes, Oxford: Blackwell.

Liebesman, D. (2015), "We Do Not Count By Identity", Australasian fournal of Philosophy, 93, 1, pp. 21-42.

Liebesman, D. (2016), "Counting as a Type of Measuring”, Philosopher's Imprint, 16, pp. 1-25.

Linnebo, O. (2014), "Plural Quantification", in The Stanford Encyclopedia of Philosophy, ed. by E. N. ZaLta, Fall 2014.

McKay, T. (2006), Plural Predication, New York: Oxford University Press.

Oliver, A. and T. Smiley (2013), Plural Logic, Oxford: Oxford University Press.

RaYo, A. (2002), "Word and Objects", Noûs, 36, 3, pp. 436-464.

Rayo, A. (2006), "Beyond Plurals", in Absolute Generality, ed. by A. Rayo and G. Uzquiano, Oxford: Oxford University Press, pp. 220-254.

Salmon, N. (1997), "Parts, Wholes, and Numbers", Philosophical Perspectives, 11, pp. 1-15.

Sider, T. (2007), "Parthood", The Philosophical Review, 116, 1, pp. 51-91.

Sider, T. (2014), "Consequences of Collapse", in Composition As Identity, ed. by D. BAXTer and A. Cotnolr, Oxford: Oxford University Press, pp. 211-221.

Spencer, J. (2016), "Counting on Strong Composition as Identity to Settle the Special Composition Question”, Erkenntnis, 82, 4, pp. 857-872.

Turner, J. (2014), "Donald Baxter's Composition as Identity", in Composition As Identity, ed. by D. BAXter and A. Cotnoir, Oxford: Oxford University Press, pp. 225-243.

Uzouiano, G. (2004), "Plurals and Simples", The Monist, 87, 3, pp. 429-451.

van Inwagen, P. (1994), "Composition as Identity”, Philosophical Perspectives, 8, pp. 207-220.

Wallace, M. (2009), Composition as Identity, PhD thesis, Chapel Hill: University of North Carolina.

Wallace, M. (2011a), “Composition as Identity: Part 1", Philosophy Compass, 6, 11, pp. 804-816.

Wallace, M. (2011b), “Composition as Identity: Part 2", Philosophy Compass, 6, 11, pp. 817-827.

YI, B.-U. (1999), “Is Mereology Ontologically Innocent?”, Philosophical Studies, 93, 2, pp. 141-160.

YI, B.-U. (2005), "The Logic and Meaning of Plurals: part I", fournal of Philosophical Logic, 34, pp. 459-506.

YI, B.-U. (2006), "The Logic and Meaning of Plurals: part II", fournal of Philosophical Logic, 35, pp. 239-288.

YI, B.-U. (2014), "Is There a Plural Object?", in Composition As Identity, ed. by D. BAXTER and A. Cotnoir, Oxford: Oxford University Press, pp. 169-191. 